\title{
A Study of an Effective Rehearsal Method in e-Learning
}

\author{
Toshiaki Horinouchi, Shinsuke Wakita, \\ Michiko Anse, and Tsutomu Tabe \\ Graduate School of Science and Engineering, Aoyama Gakuin University \\ 5-10-1, Fuchinobe, Sagamihara, Kanagawa, 229-8558, Japan \\ c5606112@cc.apyama.ac.jp, c5606138@cc.aoyama.ac.jp, \\ \{anse, tabe\} aise.aoyama.ac.jp
}

\begin{abstract}
When information is memorized, the brain rehearses it. We can preserve information over a long period of time if this rehearsal is effectively performed in studying. This research aims 1 .To clarify the effect of the rehearsal in e-learning, 2. To clarify the effective method for summary-making using a prototype system that studies structure of a stock (equity in a company). For this purpose, we 1.Compare the difference among three rehearsal methods, 2. (From result of 1) compare the results of an experiment of "Summarize" rehearsal method. As a result we find that the "Summarize" rehearsal is effective, especially when used for memorization constraints imposed in the number and types words used
\end{abstract}

Keywords: e-learning, rehearsal, Cognitive psychology, Dispersion-analysis, summarize.

\section{Introduction}

E-learning with IT technology is used as an educational tool for school education, company training, and life study with no constraints on time or place ${ }^{[3][4]}$. E-learning has yet to satisfy various demands, however, and its forms for introduction are still being development. The prohibitive costs of introducing e-learning have dissuaded educators from assuming a leadership role in disseminating e-learning, and there are still only few teaching materials available. Lee ${ }^{[2]}$ advocate a method of creating a teaching material called Instructional design (ID) from the point of system. $\mathrm{KOGO}^{[9]}$ argue that e-learning system based on learning theory can be used as a superior professor system. Action analysis study is a framework for study aiming at "Body." Situation study theory is a framework for study aiming at "Mind." Cognitive psychology, on the other hand, provides a framework for study aiming at "head." The volume of memory is a kind of index in the learning. There is no clear effective method for maintaining memory with cognitive psychology in e-learning. If we can find a method by which the memory can be effectively maintained in study, then we can promote study in e-learning as one of the new methods for making teaching materials.

This research focuses on "elaborative rehearsal" [5] in cognitive psychology. The aim is to confirm the effectiveness of the rehearsal function in the e-learning teaching material through experiments to clarify the volume of memorized information. We 
perform these experiments by constructing a prototype study support system for teaching the "structure of the stock," then we clarify an effective method for making summaries. As to be described, this research begins by demonstrating an outline of the "Rehearsal" function and the structure of the prototype learning systems that include the rehearsal function. Next, an experiment is conducted to show the effectiveness of rehearsal by comparing four prototype learning systems: a "non-rehearsal" system (a system without rehearsal), A "Questions" system (subjects solve questions), a "Summary" system (subjects make summaries), and a "questions + summary" system with rehearsal (subjects solve questions and make summaries). Lastly, an experiment is conducted to clarify a summary method by comparing four different learning systems: a "non-indication" system (a system without indication), a "limited number" system (a system with limited numbers of summary words), a "limited words" (a system with limitations of the types of summary words), and "limited numbers and types of words" (a system with limitations of the types and numbers of summary words), which the reasons for the learning system are changed based on the results of a pre-experiment.

\section{An Effective Method to Maintain the Memory}

\subsection{What Is "Rehearsal"?}

The structures of human memory are geared to "take in," "maintain," and "remember" information ${ }^{[1]}$. The mind depends on its long-term memory to fend off oblivion. Several models of maintaining memory have been developed. Fig. 1 illustrates the "multistore model" proposed by Waugh \& Norman ${ }^{[1]}$. This model proposes that the mind has two stores to retain the memory, the short-term store (STS) and long-term store (LTS). The memory kept in STS is called primary memory, while that kept in LTS is called secondary memory. Primary memory can only be maintained temporarily, for 20 seconds at most ${ }^{[8]}$. Secondary memory can he maintained forever, or until the mind slips into oblivion $\left.{ }^{[8}\right]$. The memory of which we are most aware is of course the latter, secondary memory. Information taken in is initially kept in primary memory. The information will be either forgotten or kept in the LST with rehearsal. The model also defines two types of memory rehearsals that significantly enhance the likelihood that information will remain in LTL: first, elaborative rehearsal; second, maintenance rehearsal to reinforce retention in STS ${ }^{[7]}{ }^{[10]}$. If rehearsal is effective, we can maintain the memory for a long time $\left.{ }^{[6}\right]$. Elaborative rehearsal can be further broken down into three types: "Questions" by reproduction, "Summary" by renewal, and "Questions and Summary" by reproduction and renewal. In this research we confirm the effectiveness of rehearsal as a means of elaborative rehearsal, and confirm "Summary" alone in an experiment for clarifying the summary method.

\subsection{Prototype System}

Our purpose, in making prototype systems with rehearsal, is to demonstrate the effectiveness of rehearsal methods and to clarify a summary method. Fig. 2 shows the structure and concept of the prototype system. 


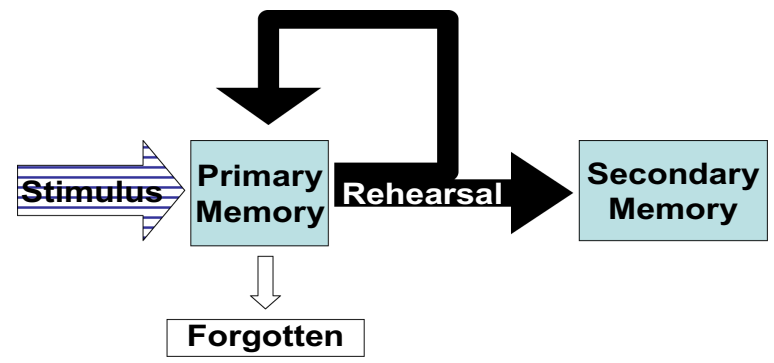

Fig. 1. Multistore model by Waugh \& Norman (1965) [1]

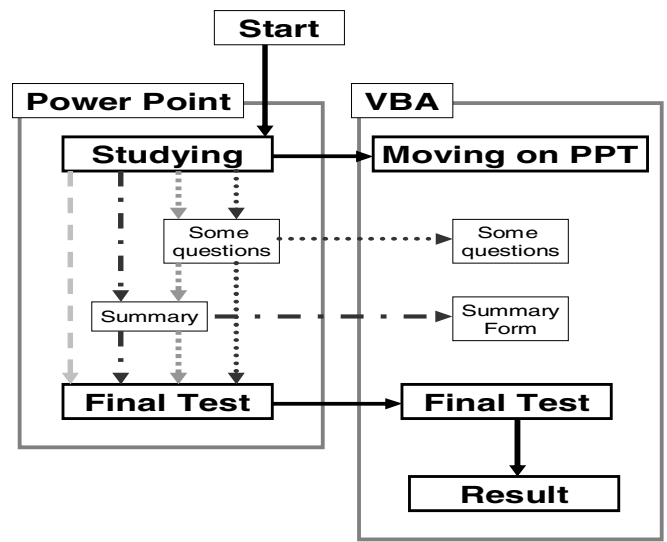

Fig. 2. Concept of the prototype system

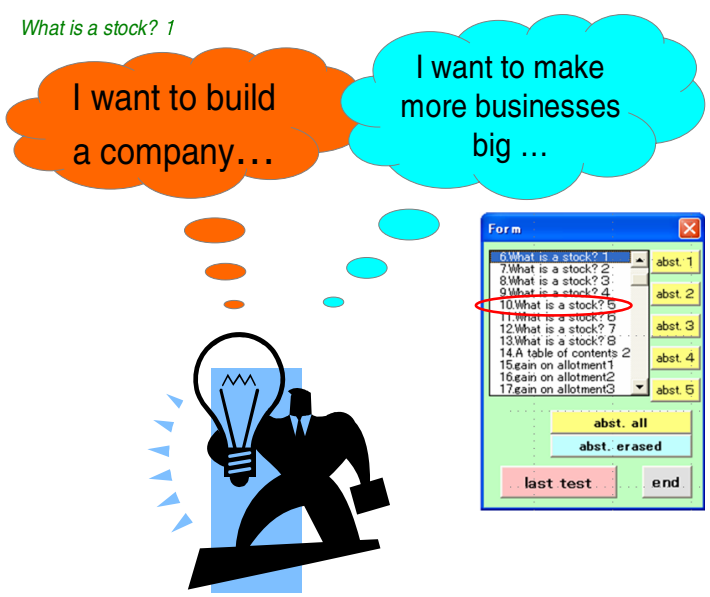

Fig. 3. Example of Power Point Content 
The prototype system is made by Microsoft Power Point (PPT) and Visual Basic for Application (VBA). The learning content is to "understand the structure of the stock." Fig. 3 shows an example of the content. This prototype system mainly consists of a study activity using PPT. It disposes of movement between slides, or shows the form of "summary" rehearsal by VBA. It is a prototype system that enables the learner to move between all of the slides with mouse clicks in order to freely inspect all of the learning material while studying. The learning content consists of five units, and a rehearsal work task is placed at the end of each unit.

\section{A Measurement Experiment of the Effective Rehearsal Method}

\subsection{A Method of the Experiment}

To measure the effectiveness of rehearsal, we ask the subjects study the prototype system and afterwards we measure the amounts of information retained in memory. The experiment is carried out in three steps: 1. Learning by prototype; 2. Questionnaire for the learner; and 3. Final Test. In the first step, the subjects asked to split up into groups of three and to study one of four prototype systems: "non-rehearsal," which has no rehearsal, "question-solving" to solve questions," "summary-making," and "question-solving and summary-making with rehearsal." In the second step, the subjects answer a questionnaire written up to ascertain whether the learners themselves noted any enhancement in the learning effect through rehearsal. In the third step, the subjects are given a final test the next day to confirm whether the learning content is memorized and to assess the amount memorized based on the rate of correct answers. The Final Test requires the subjects to answer words and phrases that come out in the prototype system in a newly generated form. It consists of 28 questions. Fig. 4 shows an example of the Final Test.

\section{We publish a stock as the evidence that you ( 1 ) some money with ( 2 ). This} money is money without ( 3 ) and calls the person who took out money a ( 4 ).

Fig. 4. Sample question from the Final Test

\subsection{A Result of the Experiment}

The bar chart in Fig. 5 plots the correct answer rate on the final test in the experiment conducted to measure the effectiveness of rehearsal.

The average of correct answer rates for the rehearsal types are 50\% for "nonrehearsal," 64\% for "question-solving," 68\% for "summary-making," and $71 \%$ for "question-solving and summary-making." Table 1, a decentralized analysis table of these correct answer rates, confirms significant differences in the effectiveness of the rehearsal types $(\mathrm{P}<5 \%)$. The multiple comparisons shown in Table 2 validate the difference between the rehearsal types. Significant differences are found in all intergroup comparisons $(\mathrm{P}<1 \%)$, except for the comparison between "Summary" and 


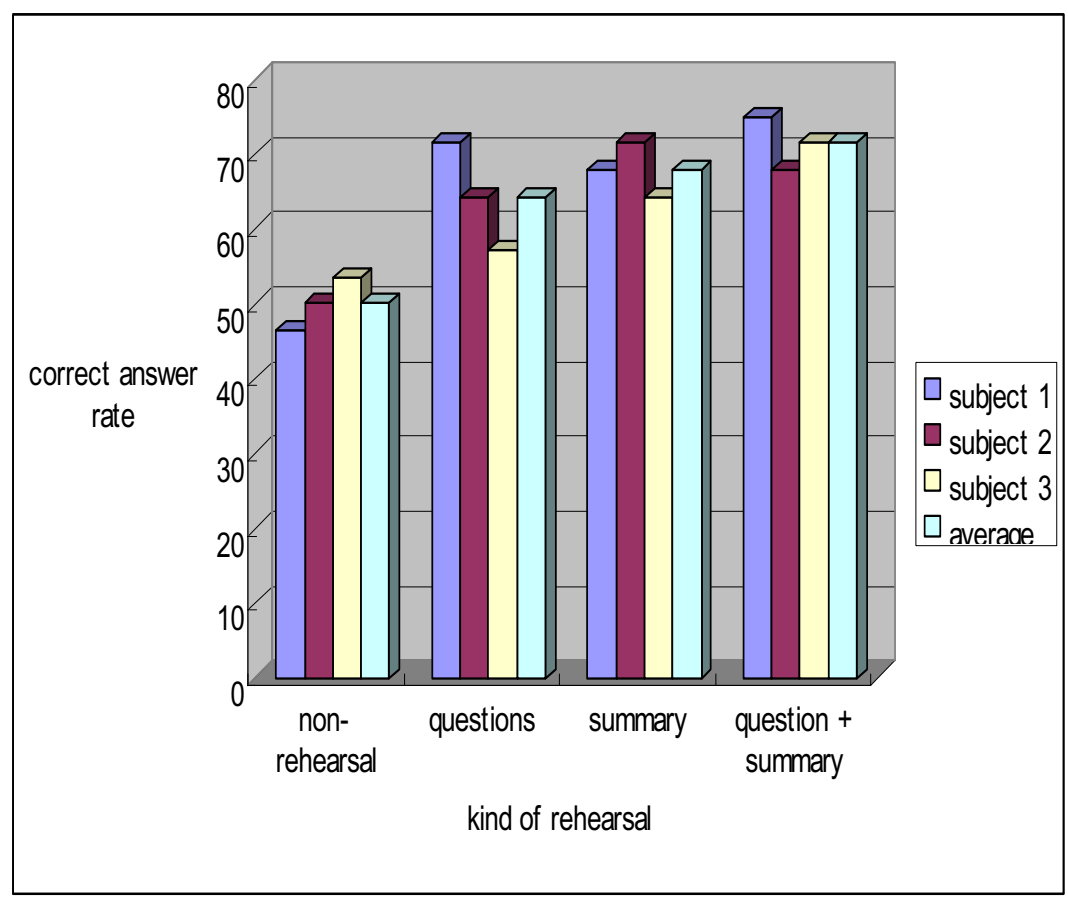

Fig. 5. Correct answer rates of rehearsal types

"Small Problem + Summary." These results demonstrate a difference between the prototype systems with and without rehearsal and show that the summary-making rehearsal can maintain the memory more effectively than the question-solving rehearsal.

To clarify the effects of the rehearsal, the questions of the final test are separated to four patterns: "questions without rehearsal," "questions with rehearsal for questionsolving," "questions with rehearsal for summary-making," and "questions with rehearsal as test in the prototype system", and compare these four patterns. Table 2 shows the results. The average of the correct answer rate rises as the level of rehearsal rises. However, no difference was seen in the correct answer rates between the problems exhibited when studying with the "Question" prototype system. The correct answer rate was question $>$ attainment level test $>$ summary, regardless of the rehearsal type. This is thought to be attributable to the difficulty of the final test.

The effects of rehearsal in this experiment are thought to be higher when the steps are carried out in an order such as "non-rehearsal < question $<$ summary $<=$ question

Table 1. Dispersion-analysis

\begin{tabular}{|c|c|r|r|c|}
\hline & $S$ & $f$ & $V$ & $F_{0}$ \\
\hline$m$ & 4270.951 & 3 & 1423.65 & $3.29107 *$ \\
\hline$e$ & 14275.11 & 33 & 432.5791 & \\
\hline$T$ & 18546.06 & 36 & \multicolumn{2}{|c}{} \\
\cline { 1 - 3 } & & &
\end{tabular}


Table 2. Multiple comparison

\begin{tabular}{|c|c|c|c|}
\hline & question & summary & $\begin{array}{r}\text { question + } \\
\text { summary }\end{array}$ \\
\hline $\begin{array}{l}\text { non- } \\
\text { rehearsal }\end{array}$ & $12.14947 * *$ & $20.25794 * *$ & $21.30952 * *$ \\
\hline question & & $8.108466 * *$ & $9.160053 * *$ \\
\hline summary & & & 1.051587 \\
\hline
\end{tabular}

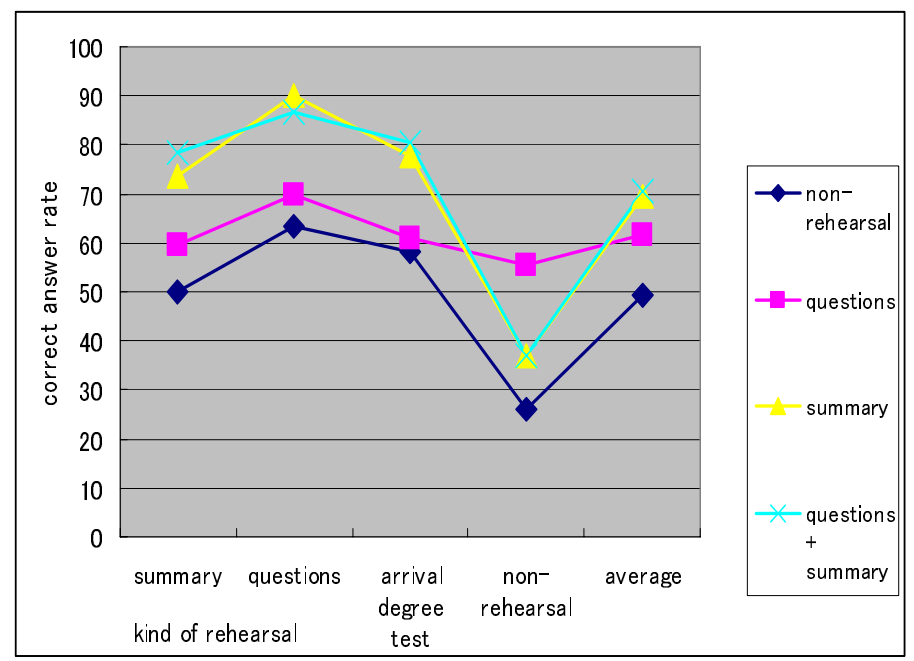

Fig. 6. Relation between correct answer rate and rehearsal

+ summary." According to the questionnaire given after the learning, all of the subjects felt that rehearsal was useful for learning and effective for enhancing memory retention.

\section{A Clarification Experiment of a Summary Method}

\subsection{A Method of the Experiment}

The pre-experiment described in chapter 3 demonstrates that "Summary" is effective as a rehearsal. Next, we examine the method used for summary-making. The experiment is carried out in the following steps: 1. A questionnaire before learning; 2 . Learning by prototype systems with "summary" rehearsal; 3. A questionnaire after learning; 4. the Final Test.

1. The questionnaire before learning checks how much knowledge the subjects have to begin with.

2. The learning by prototype systems with "summary" rehearsal finds the differences between four types of "summarize-only" rehearsal: A. "non-indication," with no 
indication; B. "limited number," with a limited number of summary words; C. "limited words," with limited types of summary words; and D. "limited number and words." The experiment is con-ducted with a prototype system that builds on these four summary methods. The summary frequency in this prototype system is corrected three times, a new group of four subjects study the prototype systems and four resulting patterns are compared.

3. A questionnaire after learning measures how subjects felt about the "summarize" activity.

4. The Final Test measures the amount of learning content retained in memory. To clarify the differences in the amounts retained, the test is organized into 40 questions expressing a number of problems. The questions which have rehearsal in the proto-type system are Nos. 1-10, 15-19, 25-34, and 38-40.

\subsection{A Result of the Experiment}

Figure 6 shows the bar chart of the correct answer rate which is in the final test of the experiment which to clarify making the summary. Each correct answer rate be-came that "non-indication" is $60 \%$, "limited number" is $58.1 \%$, "limited word" is $63.1 \%$ and "limited number and word" is $70.6 \%$.

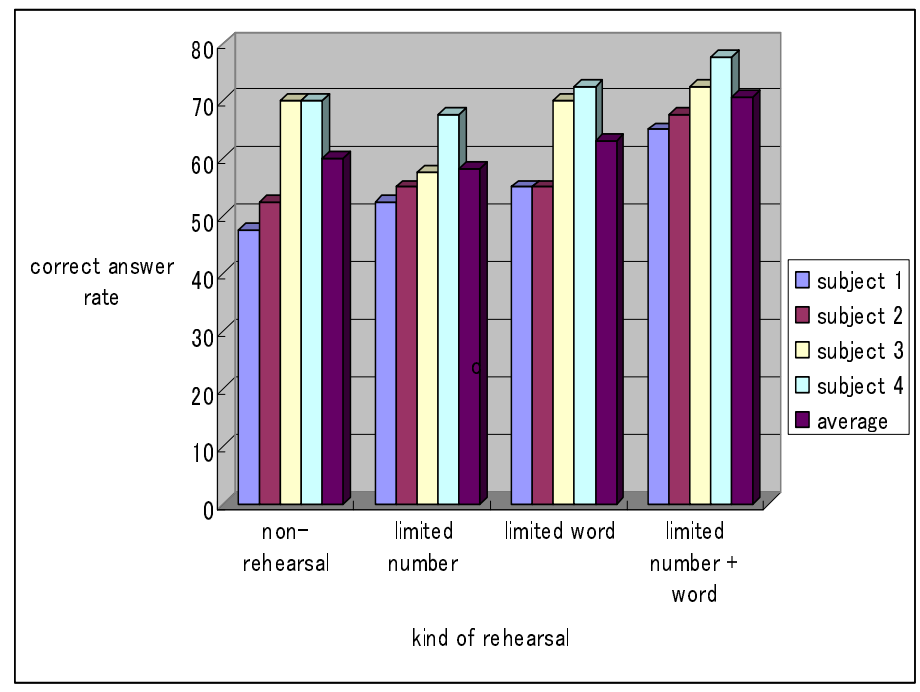

Fig. 7. Correct answer rate each summary rehearsal

Fig. 8 shows the correct answer rates from the problem to be rehearsed in the confirmation test conducted to measure the differences in the amounts of memory retention between the different summary methods. Table 3 shows the results of a decentralized analysis of these correct answer rates. Our results confirm that there are no significant differences $(\mathrm{P}<5 \%)$ among any components of the entire prototype system. Table 4 shows the results of multiple comparisons to validate whether there are differences between rehearsal types. Significant differences were found in the following 
comparisons in Table 4: the comparison between "limited number" and "limited word," the comparison between "non-indication" and "limited number and word," and the comparison between "non-indication" and "limited number and word" $(\mathrm{P}<\% 5)$. Thus, our results indicate that it is most effective to summarize specifying "limited numbers and types of words." Four-fifths $(80 \%)$ of the subjects answered that "limited words" or " limited numbers and types of words" were easier to perform as methods than "non-indication," "limited number," "limited types of words," and "limited numbers and types of words."

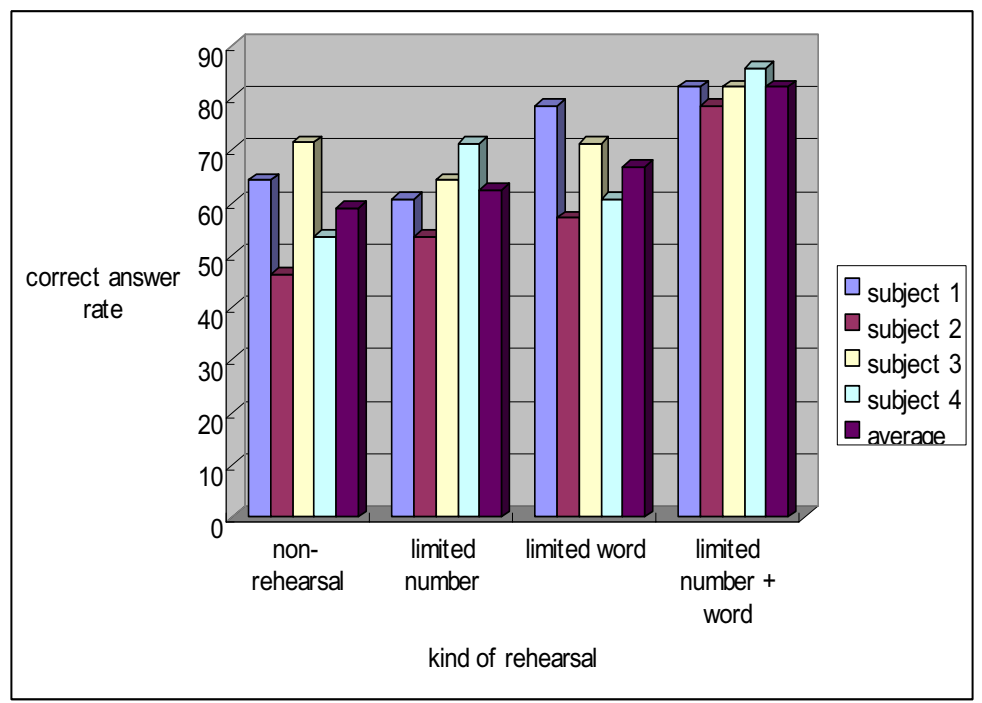

Fig. 8. Correct answer rate for summary rehearsals

Table 3. Dispersion-analysis 2

\begin{tabular}{|c|c|r|c|c|}
\hline & $\mathrm{S}$ & $\mathrm{f}$ & $\mathrm{V}$ & $\mathrm{F}_{0}$ \\
\hline $\mathrm{m}$ & 1354.432 & 3 & 451.4773 & $6.4113 *$ \\
\hline $\mathrm{e}$ & 845.0255 & 12 & 70.41879 & \\
\hline $\mathrm{T}$ & 2199.458 & 15 & & \\
\cline { 1 - 2 } & & \multicolumn{5}{|c}{}
\end{tabular}

Table 4. Multiple comparison 2

\begin{tabular}{|c|c|c|c|}
\hline & $\begin{array}{c}\text { Limited } \\
\text { word }\end{array}$ & $\begin{array}{c}\text { Limited } \\
\text { number }\end{array}$ & $\begin{array}{c}\text { Limited } \\
\text { word + } \\
\text { number }\end{array}$ \\
\hline non-rehearsal & 8.022357 & 3.558071 & $23.20093 * *$ \\
\hline limited word & & 4.464286 & 15.17857 \\
\hline limited number & & & $19.64286 * 1 \%$ \\
\hline
\end{tabular}$* \begin{aligned} & \mathrm{p}<5 \% \\
& \end{aligned}$




\section{Conclusion}

Two experiments were conducted in this research. The first experiment confirmed the effectiveness of rehearsal in e-learning. The second experiment clarified an effective summary-making method for learning. In e-learning, summary-making is effective for learning content that slips out of long-term memory, and content can be effectively memorized by specifying key words with set numbers of characters to summarize. The results of the study itself and the level of satisfaction of the learners seem to coincide. Two important problems remain to be investigated and solved henceforth. First, some e-learning systems may need to exclude students, as the learning venues may include enterprises, etc. in addition places sites of academic training. Second, the subjects who were asked to make summaries later commented that "To make summaries was surprising" and "I worried about whether it was possible to summarize it." These types of resistance need to be reduced.

\section{References}

1. Waugh, N.C., Norman, D.A.: Primary memory. psychological Renew 72, 89-104 (1965)

2. Lee, W.W., Owens, D.L.: Multimedia-Based Instructional Design

3. Marginson, Simon,: E-learning: Expanding the Bottle to Fit the Guide. International Higher Education 30, 5-8 (2003)

4. Huffaker, D.A., Calvert, S.L.: Journal of Educational Computing Research, vol. 29(3), pp. 325-334 (2003)

5. Dark, V.J., Loftus, G.R.: The Role of Rehearsal in Long-Term Memory Performance. Journal of Verbal Learning and Verbal Behavior 15(4), 479-490 (1976)

6. Meunier, G.F.: Overt Rehearsal and Long-Term Retention. Journal of Experimental Psychology 102(5), 913-914 (1974)

7. Dark, V.J., Loftus, G.R: The role of rehearsal in long-term memory performance. Journal of Verbal Learning and Verbal Behavior 15, 479-490 (1976)

8. Human Memory: The Processing of Information: GR Loftus, EF Loftus - (1976)

9. Kogo, C.: Foundations of E-Learning: Behavioral, Cognitive, Situated Learning Theories and Their Integration

10. Meunier, G.F.: Rehearsal of Individual Items in Short-Term Memory. Journal of Experimental Psychology 95(2), 465-467 (1972) 